\title{
Evaluation of Different Botanicals against Red Pumpkin Beetle, Aulacophora foveicollis Lucas Infesting Cucumber
}

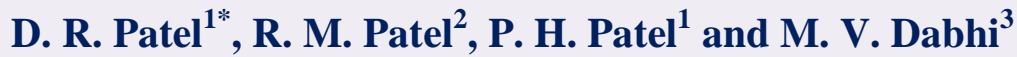

${ }^{1}$ Department of Entomology, B. A. College of Agriculture, Anand Agricultural University, Anand-388 110, Gujarat, India

${ }^{2}$ Department of Plant Protection, Krishi Vigyan Kendra, Anand Agricultural University, Devataj -387 240, Anand, India

${ }^{3}$ Sheth M. C. Polytechnic in Agriculture, Anand Agricultural University, Anand-388 110, Gujarat, India

*Corresponding author

\section{A B S T R A C T}

Keywords

Cucumber, Aulacophora

foveicollis,

Botanicals, Yield, ICBR

Article Info

Accepted:

22 January 2021

Available Online:

10 February 2021
Field experiment was conducted to determine the effectiveness of botanicals against red pumpkin beetle, Aulacophora foveicollis Lucas on cucumber in a Randomized Block Design (RBD) with 8 treatments and 3 replications during Summer, 2019 at Main Vegetable Research Station, Anand Agricultural University, Anand (Gujarat). Among botanicals evaluated against A. foveicollis on cucumber, the minimum beetle population was observed in the plot treated with azadirachtin $0.0006 \%$ and it was at par with NSKE $5 \%$ and neem oil $0.5 \%$. Of the tested botanicals, maximum fruit yield was recorded from the plot treated with azadirachtin followed by NSKE and neem oil. The maximum ICBR was registered in the treatment of azadirachtin followed by NSKE and neem leaf extract.

\section{Introduction}

Cucumber (Cucumis sativus L.) is a member of Cucurbitaceae family which is comprised of 118 genera and 825 species (Laila et al., 2015). Among various horticultural crops grown in the world, India is the second largest producer of vegetables in the world, next to China. Cucumber has been reported as a commercial cash crop in tropical and sub- tropical parts of India with an annual production of 1.14 million tonnes from an area of 78 thousand hectares and productivity is 15 tonnes per hectare (Anon., 2017). Various pests attacking the cucumber crop are fruit fly [Bactrocera cucurbitae Coq.], red pumpkin beetle [Aulacophora foveicollis Lucas], black pumpkin beetle [Aulacophora atripennis Fabricius], pumpkin caterpillar [Margaronia indica Saunders], American serpentine leaf 
miner [Liriomyza trifolii Burgess], bottle gourd moth [Sphenarches anisodactylus Walker], aphids [Aphis spp.], banded blister beetle [Zonabris postulata Tk. and Mylabris phlarata Pall.] and dudhi bugs [Nesidiocoris tenuis Reut. and Gampsocoris spp.]. Most of the insect-pests causing damage at any stage of plant growth, but some of them are serious at seedling stage viz., red pumpkin beetle, leaf miner, flea beetle, while fruit fly appears at fruiting stage (Ram et al., 2009). Red pumpkin beetle is the most serious and destructive polyphagous pest of cucurbitaceous vegetables especially cucumber, white gourd, water melon, muskmelon and sweet gourd in India (Varavdekar and Dumbre, 1992). It causes about 35 to 75 per cent damage to all cucurbits, except bitter gourd at seedling stage which needs to be re-sowing of crop. Both the adults and the grubs of red pumpkin beetle causing considerable damage to cucurbit plants during all stages of plant development (Atwal and Dhaliwal, 2002). Chemical insecticides are used as the front line defense sources against insect pests in India. However, their indiscriminate use creates a number of problems such as development of resistance against insecticides, pest resurgence and environmental hazards including residue in soil, water, vegetables and fruits. The concept of pest control with botanicals is emerging worldwide as it is eco-friendly and cost effective. Botanical pesticides are environmentally safer, unique with novel mode of action and rich source of biologically active compounds. Keeping all the above points in view, the experiment was conducted to evaluate the effectiveness of some botanicals for controlling A. foveicollis infesting cucumber crop.

\section{Materials and Methods}

In order to evaluate the efficacy of different botanicals against $A$. foveicollis on cucumber, a field experiment was carried out during summer, 2019 at Main Vegetable Research Station, Anand Agricultural University, Anand (Gujarat) in Randomized Block Design (RBD) with 8 treatments and 3 replications each having gross plot size of $7.5 \times 5.0 \mathrm{~m}$ and net plot size of $4.5 \times 3.0 \mathrm{~m}$. Cucumber variety Gujarat Cucumber-1 was sown at spacing of $1.5 \times 1.0 \mathrm{~m}$ on $2^{\text {nd }}$ March, 2019.

Treatments were used: $\mathrm{T}_{1}=$ Neem Seed Kernel Extract $5 \%, \mathrm{~T}_{2}=$ Neem leaf extract $10 \%, \mathrm{~T}_{3}=$ Ardusi leaf extract 5\%, $\mathrm{T}_{4}=$ Neem Oil $0.5 \%$, $\mathrm{T}_{5}=$ Garlic bulb extract 5\%, $\mathrm{T}_{6}=$ Ginger rhizome extract $5 \%, \mathrm{~T}_{7}=$ Azadirachtin $0.15 \mathrm{EC}$ $0.0006 \%$ and $\mathrm{T}_{8}=$ control. Cucumber crop was raised by adopting all recommended agronomical practices except insecticide application.

The first spray of respective botanicals was sprayed after the appearance of pest. The second spray was applied after 10 days of first spray. Spray fluid was applied by using manually operated knapsack sprayer fitted with hollow cone nozzle. For the purpose of recording the observations, five plants were randomly selected from each net plot. Observations on red pumpkin beetle were recorded prior as well as 3, 5, 7 and 10 days after each spray.

The fruit yield was recorded picking wise from each plot. Then the plot-wise yield obtained was converted into $\mathrm{q} / \mathrm{ha}$. For determine the increase in yield and avoidable losses, following formula given by Khosla (1977) were used:
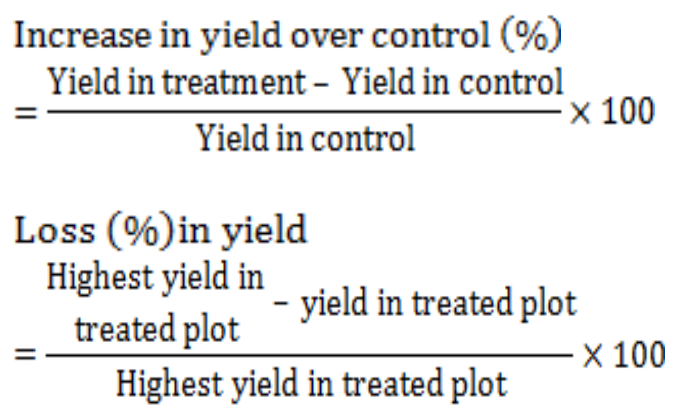


\section{Results and Discussion}

The data on red pumpkin beetle population before spraying of botanicals showed nonsignificant differences among different treatments which indicated homogenous distribution of pest in the experimental plot (Table 1). All the evaluated botanicals were found significantly superior over control up-to 10 days of spray.

The data on pooled over periods of first spray differ significantly to each other. Minimum population of red pumpkin beetle was recorded in plots treated with azadirachtin 0.0006 per cent ( 2.57 beetles/plant) and it was found at par with NSKE 5 per cent (3.09 beetles/plant). These two treatments were found significantly superior to rest of the treatments. Neem oil 0.5 per cent (3.48 beetles/plant) and garlic bulb extract 5 per cent (4.13 beetles/plant) were found effective with lower beetle population and remained as next effective treatments in controlling the pest. The plot treated with ginger rhizome extract 5 per cent recorded maximum (4.41 beetles/plant) population of red pumpkin beetle and it was at par with neem leaf extract 10 per cent ( 4.22 beetles/plant) and ardusi leaf extract 5 per cent (4.31 beetles/plant) and proved as less effective.

The data on pooled over periods of second spray revealed that azadirachtin 0.0006 per cent recorded significantly lowest (1.17 beetles/plant) beetle population and it was at par with NSKE 5 per cent (1.36 beetles/plant).

Neem oil 0.5 per cent recorded (1.76 beetles/plant) population of red pumpkin beetle and stood next effective treatment. Neem leaf extract 10 per cent, garlic bulb extract 5 per cent and ardusi leaf extract 5 per cent $(2.25,2.27$ and 2.39 beetles/plant) were statistically equally effective in controlling the beetle population and stood at par with each other. Of the tested botanicals, ginger rhizome extract 5 per cent found least effective by recording the highest beetle population (2.75 beetles/plant).

Overall, the data on pooled over sprays showed that significantly the lowest beetle population was recorded in the plot treated with azadirachtin 0.0006 per cent (1.81 beetles/plant). NSKE 5 per cent $(2.16$ beetles/plant) and neem oil 0.5 per cent (2.56 beetles/plant) remained as next effective treatment in suppressing the beetle population and both were differed significantly from each other. The highest population was recorded in plot ginger rhizome extract 5 per cent (3.54 beetles/plant) and it was found at par with garlic bulb extract 5 per cent $(3.15$ beetles/plant), neem leaf extract 10 per cent (3.19 beetles/plant) and ardusi leaf extract 5 per cent (3.30 beetles/plant).

\section{Yield}

The data (Table 2) on yield showed higher fruit yield in all the treated plots than untreated plot. The maximum yield (122.58 $\mathrm{q} / \mathrm{ha}$ ) recorded in azadirachtin 0.0006 per cent and at par with NSKE 5 per cent (117.07 q/ha). Minimum yield (87.29 q/ha) was obtained in ginger rhizome extract 5 per cent which was at par with ardusi leaf extract 5 per cent $(93.64 \mathrm{q} / \mathrm{ha})$ and neem leaf extract 10 per cent $(98.58 \mathrm{q} / \mathrm{ha})$.

Maximum (70.04\%) increase in yield over control was recorded in plot treated with azadirachtin 0.0006 per cent followed by NSKE 5 per cent $(62.40 \%)$ and neem oil 0.5 per cent (54.25\%). Minimum (21.08\%) increase in yield was recorded in plot treated with ginger rhizome extract 5 per cent followed by ardusi leaf extract 5 per cent (29.90\%), neem leaf extract 10 per cent (36.74\%) and garlic bulb extract 5 per cent $(49.31 \%)$. 
Table.1 Effectiveness of different botanicals against A. foveicollis on cucumber (Pooled over periods and sprays)

\begin{tabular}{|c|c|c|c|c|c|c|c|c|c|c|c|c|c|c|}
\hline \multirow{3}{*}{$\begin{array}{l}\text { Tr. } \\
\text { No. }\end{array}$} & \multirow[t]{3}{*}{ Treatments } & \multirow{3}{*}{$\begin{array}{l}\text { Conc. } \\
(\%)\end{array}$} & \multicolumn{12}{|c|}{ No. of red pumpkin beetles/plant at indicated days after spray } \\
\hline & & & \multirow{2}{*}{$\begin{array}{l}\text { Before } \\
\text { spray }\end{array}$} & \multicolumn{5}{|c|}{ First } & \multicolumn{5}{|c|}{ Second } & \multirow{2}{*}{$\begin{array}{c}\text { Pooled ove } \\
\text { sprays }\end{array}$} \\
\hline & & & & 3 & 5 & 7 & 10 & Pooled & 3 & 5 & 7 & 10 & Pooled & \\
\hline 1 & Neem seed kernel extract & 5.0 & $\begin{array}{c}2.50 \\
(5.77)\end{array}$ & $\begin{array}{c}2.07 \mathrm{ab} \\
(3.78)\end{array}$ & $\begin{array}{l}1.90 \mathrm{ab} \\
(3.12)\end{array}$ & $\begin{array}{l}1.73 \mathrm{ab} \\
(2.49)\end{array}$ & $\begin{array}{l}1.88 \mathrm{a} \\
(3.03)\end{array}$ & $\begin{array}{l}1.90 \mathrm{ab} \\
(3.09)\end{array}$ & $\begin{array}{l}1.56 \mathrm{ab} \\
(1.93)\end{array}$ & $\begin{array}{l}1.40 \mathrm{ab} \\
(1.47)\end{array}$ & $\begin{array}{l}1.32 \mathrm{ab} \\
(1.25)\end{array}$ & $\begin{array}{l}1.16 \mathrm{a} \\
(0.85)\end{array}$ & $\begin{array}{l}1.36 \mathrm{a} \\
(1.36)\end{array}$ & $\begin{array}{l}1.63 b \\
(2.16)\end{array}$ \\
\hline 2 & Neem leaf extract & 10.0 & $\begin{array}{c}2.45 \\
(5.50)\end{array}$ & $\begin{array}{c}2.38 \mathrm{bcd} \\
(5.18)\end{array}$ & $\begin{array}{l}2.21 b c \\
(4.38)\end{array}$ & $\begin{array}{c}2.02 \mathrm{bc} \\
(3.57)\end{array}$ & $\begin{array}{l}2.08 \mathrm{ab} \\
(3.84)\end{array}$ & $\begin{array}{l}2.17 d \\
(4.22)\end{array}$ & $\begin{array}{c}1.92 \mathrm{cde} \\
(3.19)\end{array}$ & $\begin{array}{l}1.78 \mathrm{~cd} \\
(2.66)\end{array}$ & $\begin{array}{c}1.51 \mathrm{abc} \\
(1.78)\end{array}$ & $\begin{array}{c}1.43 \mathrm{abc} \\
(1.54)\end{array}$ & $\begin{array}{l}1.66 \mathrm{c} \\
(2.25)\end{array}$ & $\begin{array}{l}1.92 \mathrm{~d} \\
(3.19)\end{array}$ \\
\hline 3 & Ardusi leaf extract & 5.0 & $\begin{array}{c}2.49 \\
(5.68)\end{array}$ & $\begin{array}{l}2.41 b c \\
(5.32)\end{array}$ & $\begin{array}{l}2.24 b c \\
(4.52)\end{array}$ & $\begin{array}{c}2.03 \mathrm{bc} \\
(3.61)\end{array}$ & $\begin{array}{c}2.10 \mathrm{ab} \\
(3.90)\end{array}$ & $\begin{array}{l}2.19 \mathrm{~d} \\
(4.31)\end{array}$ & $\begin{array}{l}1.99 \mathrm{de} \\
(3.45)\end{array}$ & $\begin{array}{l}1.82 \mathrm{~d} \\
(2.80)\end{array}$ & $\begin{array}{c}1.53 \mathrm{abc} \\
(1.85)\end{array}$ & $\begin{array}{c}1.47 \mathrm{abc} \\
(1.65)\end{array}$ & $\begin{array}{l}1.70 \mathrm{~cd} \\
(2.39)\end{array}$ & $\begin{array}{l}1.95 \mathrm{~d} \\
(3.30)\end{array}$ \\
\hline 4 & Neem Oil & 0.5 & $\begin{array}{c}2.43 \\
(5.40)\end{array}$ & $\begin{array}{c}2.22 \mathrm{abc} \\
(4.44)\end{array}$ & $\begin{array}{l}2.02 b c \\
(3.58)\end{array}$ & $\begin{array}{l}1.81 \mathrm{ab} \\
(2.76)\end{array}$ & $\begin{array}{l}1.93 \mathrm{a} \\
(3.21)\end{array}$ & $\begin{array}{l}1.99 \mathrm{bc} \\
(3.48)\end{array}$ & $\begin{array}{c}1.70 \mathrm{abc} \\
(2.39)\end{array}$ & $\begin{array}{l}1.57 b c \\
(1.98)\end{array}$ & $\begin{array}{l}1.39 \mathrm{ab} \\
(1.44)\end{array}$ & $\begin{array}{l}1.35 \mathrm{ab} \\
(1.32)\end{array}$ & $\begin{array}{l}1.50 \mathrm{~b} \\
(1.76)\end{array}$ & $\begin{array}{l}1.75 c \\
(2.56)\end{array}$ \\
\hline 5 & Garlic bulb extract & 5.0 & $\begin{array}{c}2.50 \\
(5.73)\end{array}$ & $\begin{array}{l}2.47 \mathrm{bc} \\
(5.58)\end{array}$ & $\begin{array}{l}2.18 b c \\
(4.24)\end{array}$ & $\begin{array}{l}1.93 \mathrm{ab} \\
(3.24)\end{array}$ & $\begin{array}{l}2.03 \mathrm{a} \\
(3.63)\end{array}$ & $\begin{array}{l}2.15 \mathrm{~cd} \\
(4.13)\end{array}$ & $\begin{array}{c}1.80 \mathrm{bcd} \\
(2.73)\end{array}$ & $\begin{array}{l}1.68 \mathrm{~cd} \\
(2.32)\end{array}$ & $\begin{array}{c}1.57 \mathrm{abc} \\
(1.98)\end{array}$ & $\begin{array}{l}1.60 \mathrm{bc} \\
(2.07)\end{array}$ & $\begin{array}{l}1.66 \mathrm{~cd} \\
(2.27)\end{array}$ & $\begin{array}{l}1.91 \mathrm{~d} \\
(3.15)\end{array}$ \\
\hline 6 & Ginger rhizome extract & 5.0 & $\begin{array}{c}2.47 \\
(5.67)\end{array}$ & $\begin{array}{l}2.41 b c \\
(5.32)\end{array}$ & $\begin{array}{l}2.25 \mathrm{c} \\
(4.56)\end{array}$ & $\begin{array}{l}2.09 \mathrm{bc} \\
(3.87)\end{array}$ & $\begin{array}{l}2.11 \mathrm{ab} \\
(3.97)\end{array}$ & $\begin{array}{l}2.22 \mathrm{~d} \\
(4.41)\end{array}$ & $\begin{array}{l}2.11 \mathrm{e} \\
(3.97)\end{array}$ & $\begin{array}{l}1.81 \mathrm{~cd} \\
(2.76)\end{array}$ & $\begin{array}{l}1.65 b c \\
(2.23)\end{array}$ & $\begin{array}{l}1.64 b c \\
(2.19)\end{array}$ & $\begin{array}{l}1.80 \mathrm{~d} \\
(2.75)\end{array}$ & $\begin{array}{l}2.01 \mathrm{~d} \\
(3.54)\end{array}$ \\
\hline 7 & Azadirachtin $0.15 \mathrm{EC}$ & 0.0006 & $\begin{array}{c}2.47 \\
(5.60)\end{array}$ & $\begin{array}{l}2.03 \mathrm{a} \\
(3.63)\end{array}$ & $\begin{array}{l}1.63 \mathrm{a} \\
(2.17)\end{array}$ & $\begin{array}{l}1.57 \mathrm{a} \\
(1.95)\end{array}$ & $\begin{array}{l}1.78 \mathrm{a} \\
(2.67)\end{array}$ & $\begin{array}{l}1.75 \mathrm{a} \\
(2.57)\end{array}$ & $\begin{array}{l}1.48 \mathrm{a} \\
(1.70)\end{array}$ & $\begin{array}{l}1.26 \mathrm{a} \\
(1.10)\end{array}$ & $\begin{array}{l}1.26 \mathrm{a} \\
(1.09)\end{array}$ & $\begin{array}{l}1.16 \mathrm{a} \\
(0.85)\end{array}$ & $\begin{array}{l}1.29 \mathrm{a} \\
(1.17)\end{array}$ & $\begin{array}{l}1.52 \mathrm{a} \\
(1.81)\end{array}$ \\
\hline 8 & Control & - & $\begin{array}{c}2.48 \\
(5.63)\end{array}$ & $\begin{array}{l}2.57 d \\
(6.12)\end{array}$ & $\begin{array}{l}2.34 \mathrm{c} \\
(4.99)\end{array}$ & $\begin{array}{l}2.40 \mathrm{c} \\
(5.26)\end{array}$ & $\begin{array}{l}2.45 \mathrm{~b} \\
(5.54)\end{array}$ & $\begin{array}{l}2.44 \mathrm{e} \\
(5.47)\end{array}$ & $\begin{array}{c}2.40 \mathrm{f} \\
(5.28)\end{array}$ & $\begin{array}{l}2.10 \mathrm{e} \\
(3.91)\end{array}$ & $\begin{array}{l}1.82 \mathrm{c} \\
(2.80)\end{array}$ & $\begin{array}{l}1.75 \mathrm{c} \\
(2.55)\end{array}$ & $\begin{array}{l}2.02 \mathrm{e} \\
(3.57)\end{array}$ & $\begin{array}{l}2.23 \mathrm{e} \\
(4.47)\end{array}$ \\
\hline & S. Em. $\pm \quad T$ & & 0.15 & 0.10 & 0.10 & 0.13 & 0.12 & 0.06 & 0.08 & 0.07 & 0.10 & 0.10 & 0.04 & 0.04 \\
\hline & $\mathbf{P}$ & & - & - & - & - & - & 0.04 & - & - & - & - & 0.03 & 0.02 \\
\hline & $\mathbf{S}$ & & - & - & - & - & - & - & - & - & - & - & - & 0.02 \\
\hline & $\mathbf{T} \times \mathbf{P}$ & & - & - & - & - & - & 0.11 & - & - & - & - & 0.09 & 0.07 \\
\hline & $\mathrm{T} \times \mathrm{S}$ & & - & - & - & - & - & - & - & - & - & - & - & 0.05 \\
\hline & $\mathrm{P} \times \mathrm{S}$ & & - & - & - & - & - & - & - & - & - & - & - & 0.04 \\
\hline & $\mathrm{T} \times \mathrm{P} \times \mathrm{S}$ & & - & - & - & - & - & - & - & - & - & - & - & 0.10 \\
\hline & C. D. at $5 \%$ & & NS & Sig. & Sig. & Sig. & Sig. & Sig. & Sig. & Sig. & Sig. & Sig. & Sig. & Sig. \\
\hline & C. V. \% & & 10.18 & 7.10 & 8.40 & 12.00 & 9.85 & 9.30 & 7.28 & 7.71 & 11.85 & 12.11 & 9.28 & 9.37 \\
\hline
\end{tabular}

Note: 1. Figures in parenthesis are retransformed values; those outside are $\sqrt{x+0.5}$ transformed values

2. Treatment mean(s) with the letter(s) in common are not significant by DNMRT at $5 \%$ level of significance

3. Significant parameters and its interactions: T, P, S, P x S, where $P=$ Period and $S=S$ Spray 
Table.2 Effectiveness of different botanicals on fruit yield of cucumber

\begin{tabular}{|c|c|c|c|c|c|}
\hline $\begin{array}{l}\text { Tr. } \\
\text { No. }\end{array}$ & Treatments & $\begin{array}{l}\text { Conc. } \\
(\%)\end{array}$ & $\begin{array}{l}\text { Yield } \\
\text { (q/ha) }\end{array}$ & $\begin{array}{l}\text { Increase in yield } \\
\text { over control }(\%)\end{array}$ & $\begin{array}{c}\text { Avoidable losses } \\
(\%)\end{array}$ \\
\hline $\mathbf{T}_{1}$ & Neem seed kernel extract & 5.0 & $117.07 \mathrm{ab}$ & 62.40 & 4.50 \\
\hline $\mathbf{T}_{2}$ & Neem leaf extract & 10.0 & $98.58 \mathrm{bcd}$ & 36.74 & 19.58 \\
\hline $\mathbf{T}_{3}$ & Ardusi leaf extract & 5.0 & $93.64 \mathrm{~cd}$ & 29.90 & 23.61 \\
\hline $\mathbf{T}_{4}$ & Neem Oil & 0.5 & $111.20 \mathrm{abc}$ & 54.25 & 9.28 \\
\hline $\mathbf{T}_{5}$ & Garlic bulb extract & 5.0 & $107.64 \mathrm{abc}$ & 49.31 & 12.19 \\
\hline $\mathbf{T}_{6}$ & Ginger rhizome extract & 5.0 & $87.29 \mathrm{de}$ & 21.08 & 28.79 \\
\hline $\mathbf{T}_{7}$ & Azadirachtin $0.15 \mathrm{EC}$ & 0.0006 & $122.58 \mathrm{a}$ & 70.04 & 00.00 \\
\hline $\mathbf{T}_{8}$ & Control & - & $72.09 \mathrm{e}$ & - & 41.19 \\
\hline \multicolumn{2}{|r|}{ S. Em. \pm} & & 6.07 & - & - \\
\hline \multicolumn{2}{|r|}{ C. V. $(\%)$} & & 10.38 & - & - \\
\hline
\end{tabular}


Table.3 Economics of different botanicals evaluated against $A$. foveicollis on cucumber

\begin{tabular}{|c|c|c|c|c|c|c|c|c|c|c|c|c|}
\hline $\begin{array}{l}\text { Tr } \\
\dot{\mathbf{N}} \\
\text { o. }\end{array}$ & Botanicals & $\begin{array}{c}\text { Conc. } \\
(\%)\end{array}$ & $\begin{array}{l}\text { Quantity of } \\
\text { botanicals } \\
\text { required for } \\
2 \text { sprays } \\
\text { (kg or l/ha) }\end{array}$ & $\begin{array}{c}\text { Price of } \\
\text { botanical } \\
\text { s } \\
\text { (₹ /litre } \\
\text { or kg) }\end{array}$ & $\begin{array}{c}\text { Cost of } \\
\text { botanical } \\
\text { S } \\
\text { (₹ /ha) }\end{array}$ & $\begin{array}{c}\text { Labo } \\
\text { ur } \\
\text { cost } \\
(₹ / h a \\
\text { ) }\end{array}$ & $\begin{array}{l}\text { Total } \\
\text { cost } \\
(₹ / h a)\end{array}$ & $\begin{array}{l}\text { Yield } \\
\text { (q/ha) }\end{array}$ & $\begin{array}{l}\text { Net } \\
\text { gain } \\
\text { over } \\
\text { control } \\
\text { (q/ha) }\end{array}$ & $\begin{array}{c}\text { Realizatio } \\
\text { n } \\
\text { (₹ /ha) }\end{array}$ & $\begin{array}{l}\text { Net } \\
\text { profi } \\
\text { t } \\
(₹ / h \\
\text { a) }\end{array}$ & ICBR \\
\hline $\mathbf{T}_{1}$ & $\begin{array}{c}\text { Neem seed kernel } \\
\text { extract }\end{array}$ & 5.0 & 50 & 20 & 1000 & 980 & 1980 & 117.07 & 44.98 & 44980 & $\begin{array}{c}4300 \\
0\end{array}$ & $\begin{array}{c}1: \\
22.71\end{array}$ \\
\hline $\mathbf{T}_{2}$ & Neem leaf extract & 10.0 & 100 & 4 & 400 & 980 & 1380 & 98.58 & 26.49 & 26490 & $\begin{array}{c}2511 \\
0\end{array}$ & $\begin{array}{c}1: \\
19.19\end{array}$ \\
\hline $\mathbf{T}_{3}$ & Ardusi leaf extract & 5.0 & 50 & 4 & 200 & 980 & 1180 & 93.64 & 21.55 & 21550 & $\begin{array}{c}2037 \\
0\end{array}$ & $\begin{array}{c}1: \\
18.26\end{array}$ \\
\hline $\mathbf{T}_{4}$ & Neem Oil & 0.5 & 5 & 400 & 2000 & 980 & 2980 & 111.20 & 39.11 & 39110 & $\begin{array}{c}3613 \\
0\end{array}$ & $\begin{array}{c}1: \\
13.12\end{array}$ \\
\hline $\mathbf{T}_{5}$ & Garlic bulb extract & 5.0 & 50 & 50 & 2500 & 980 & 3480 & 107.64 & 35.55 & 35550 & $\begin{array}{c}3207 \\
0\end{array}$ & $\begin{array}{c}1: \\
10.21\end{array}$ \\
\hline $\mathbf{T}_{6}$ & Ginger rhizome extract & 5.0 & 50 & 60 & 3000 & 980 & 3980 & 87.29 & 15.20 & 15200 & $\begin{array}{c}1122 \\
0\end{array}$ & $1: 3.81$ \\
\hline $\mathbf{T}_{7}$ & Azadirachtin $0.15 \mathrm{EC}$ & $\begin{array}{c}0.000 \\
6\end{array}$ & 4 & 300 & 1200 & 980 & 2180 & 122.58 & 50.49 & 50490 & $\begin{array}{c}4831 \\
0\end{array}$ & $\begin{array}{c}1: \\
23.16\end{array}$ \\
\hline $\mathbf{T}_{8}$ & Control & - & - & - & - & - & - & 72.09 & - & - & - & - \\
\hline Not & $\begin{array}{l}\text { 1. Labour charges @ ₹ } \\
\text { 2. For } 2 \text { spray @ } 2 * 490 \\
\text { 3. Price of cucumber fr }\end{array}$ & $\begin{array}{l}2+₹ \\
80 ₹ / 1 \\
1000\end{array}$ & 78/- per & Total & 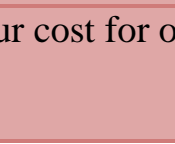 & & & 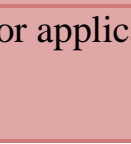 & 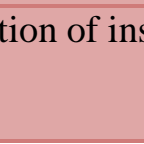 & cides & & \\
\hline
\end{tabular}


Maximum (41.19\%) avoidable losses were obtained in control plot, whereas minimum $(4.50 \%)$ losses was obtained in NSKE 5 per cent. The details of Incremental Cost Benefit Ratio (ICBR) calculated for different botanicals are presented in Table 3.

Data revealed that maximum (50490 ₹/ha) realization was obtained in case of azadirachtin 0.0006 per cent treated plots followed by NSKE 5 per cent (44980 ₹ /ha) and neem oil 0.5 per cent (39110 ₹/ha). While, minimum (15200 ₹/ha) realization was obtained from the ginger rhizome extract 5 per cet.

Maximum (1:23.16) ICBR registered in azadirachtin 0.0006 per cent followed by NSKE 5 per cent $(1: 22.71)$ and neem leaf extract 10 per cent $(1: 19.19)$. While, minimum (1:3.81) ICBR was recorded in the plot treated with ginger rhizome extract 5 per cent.

Gujar and Mehrotra (1988) reported that NSKE 0.01 per cent and neem oil 0.4 per cent reduced the feeding activity of red pumpkin beetle upto 50 per cent. Rajak and Singh (2002) reported that leaf powder of neem (Azadirachta indica) was most effective against the red pumpkin beetle followed by balcayen (Melia azadirach). Lakshmi et al., (2005) revealed that spray of nimbecidine $(28.66 \%)$ was found most effective in reducing the beetle population as well as in reducing the leaf damage.

Rashid et al., (2016) who noted that spraying with NSKE 5\% recorded the minimum (4.31 beetles/plant) population followed by Moringa olefera (4.89 beetles/plant). Chowdhury and Talukder (2019) revealed that spray of neem oil and malathion recorded the minimum (1.26 and 1.30 beetles/plant, respectively) number of beetle population followed by mahogoni seeds (1.80 beetles/plant) and pyrethrum flower (2.12 beetles/plant).

\section{Acknowledgement}

We are sincerely thankful to Director of Research and Dean PG Studies, Principal and Dean (Agri.) for conducting this research work. We are also thankful to Dr. R. R. Acharya, Head and Research Scientist (Veg.) and Dr. M. M. Pandya, Assistant Research Scientist, Main Vegetables Research Station for providing experimental farm and other resources.

\section{References}

Anonymous (2017). Horticultural statistics at a Glance, Department of Agriculture, Cooperation \& Farmers Welfare, GOI, pp. 204.

Atwal, A. S. and Dhaliwal, G. S. (2002). Agricultural Pests of South Asia and their management. Kalyani Publishers, Ludhiana, New Delhi, India, pp. 262263, 487.

Chowdhury, S. A. R. and Talukder, J. (2019). Practice of botanical pesticides for sustainable and safe vegetables production in Bangladesh. Bangladesh Journal of Environmental Science, 37, 82-87.

Gujar, G. T. and Mehrotra, K. N. (1988). Biological activity of neem against the red pumpkin beetle, Aulacophora foveicollis. Journal of Phytoparasitica, 16 (4): 293-302.

Khosla, R. K. (1977). Techniques for assessment of losses due to pests and diseases of rice. Indian Journal of Agricultural Science, 47 (4): 171-174.

Laila K., Shah, M. and Usman, A. (2015). Host preference of red pumpkin beetle (Aulacophora foveicollis L.) (Chrysomelidae: Coleoptera) among different cucurbits. Journal of Entomology and Zoology Studies, 3 (2): 100-104. 
Lakshmi, M. V., Rao, G. R. and Rao, P. A. (2005). Efficacy of different insecticides against red pumpkin beetle, Raphidopalpa foveicollis Lucas on pumpkin, Cucurbita maxima Duchesne. Journal of Applied Zoological Researches, 16(1): 73-74.

Rajak, D. C. and Singh, H. M. (2002). Comparative efficacy of pesticides against red pumpkin beetle, $A$. foveicollis on muskmelon. Annals of Plant Protection Science, 10 (1): 147148.

Ram, H. H., Prasad, L., Singh, O. K., Yadav, R. S. and Singh, B. (2009). Screening of cucurbit germplasm against insectpests and diseases under natural conditions. Society for Recent Development Agriculture, 9 (2): 229234.

Rashid, M. A., Naeem, M., Tariq, M., Khan, M. A. and Shaheen, F. A. (2016). Population and infestation assessment of red pumpkin beetle (Aulacophora foveicollis Lucas) and management using botanicals. Journal of Entomology and Zoology Studies, 4 (4): 1072-1075.

Varavdekar, Y. R. and Dumbre, R. B. (1992). Laboratory studies on bionomics and host preference of Raphidopalpa spp. in Maharashtra. Journal of Maharashtra Agricultural University, 17, 254-257.

\section{How to cite this article:}

Patel, D. R., R. M. Patel, P. H. Patel and Dabhi, M. V. 2021. Evaluation of Different Botanicals against Red Pumpkin Beetle, Aulacophora foveicollis Lucas Infesting Cucumber. Int.J.Curr.Microbiol.App.Sci. 10(02): 3133-3140.

doi: https://doi.org/10.20546/ijcmas.2021.1002.343 